\title{
ON EFFECTS OF STOCHASTIC REGULARIZATION FOR THE PRESSURELESS GAS DYNAMICS
}

\author{
ANASTASIA KORSHUNOVA $^{1}$, OLGA ROZANOVA ${ }^{1}$
}

\begin{abstract}
We extend our result of 1$]$ and show that one can associate with the stochastically perturbed non-viscid Burgers equation a system of viscous balance laws. The Cauchy data for the Burgers equation generates the data for this system. Till the moment of the shock formation in the solution to the Burgers equation the above system of viscous balance laws can be reduced to the pressureless gas dynamics system (in a limit as the parameters of perturbation tend to zero). If the solution to the Burgers equation contains shocks, the limit system is equivalent to the system with a specific pressure, in some sense analogous to the pressure of barotropic monoatomic gas.
\end{abstract}

\section{Stochastic perturbation of the Burgers EQUATION}

Let us consider the Cauchy problem for the non-viscous Burgers equation:

$$
\partial_{t} u+(u, \nabla) u=0, t>0, \quad u(x, 0)=u_{0}(x) \in C^{1}\left(\mathbb{R}^{n}\right) \cap C_{b}\left(\mathbb{R}^{n}\right) .
$$

Here $u(x, t)=\left(u_{1}, \ldots, u_{n}\right)(x, t)$ is a vector-function $\mathbb{R}^{n+1} \rightarrow \mathbb{R}^{n}$.

We associate with (1.1) the following system of stochastic differential equations:

$$
\begin{gathered}
d X_{k}(t)=U_{k}(t) d t+\sigma_{1} d\left(W_{k}^{1}\right)_{t}, \quad d U_{k}(t)=\sigma_{2} d\left(W_{k}^{2}\right)_{t}, \quad k=1, \ldots, n, \\
X(0)=x, \quad U(0)=u, \quad t>0,
\end{gathered}
$$

where $X(t)$ and $U(t)$ are considered as random variables with given initial distributions, $(X(t), U(t))$ runs in the phase space $\mathbb{R}^{n} \times \mathbb{R}^{n}, \sigma_{1}$ and $\sigma_{2}$ are nonnegative constants such that $|\sigma| \neq 0\left(\sigma=\left(\sigma_{1}, \sigma_{2}\right)\right)$

1991 Mathematics Subject Classification. 35L65; 35L67.

Key words and phrases. pressureless gas, non-viscous Burgers equation, stochastic perturbation, non-interacting particles, sticky particles, $\delta$ - singularity, Hugoniot conditions, spurious pressure, monoatomic gas.

Supported by DFG 436 RUS 113/823/0-1 (O.R.) and the special program of the Ministry of Education of the Russian Federation "The development of scientific potential of the Higher School", project 2.1.1/1399 (A.K. and O.R.). 
and $\left(W^{i}\right)_{t}=\left(W_{1}^{i}, \ldots, W_{n}^{i}\right)_{t}, i=1,2$, are independent $n$ - dimensional Brownian motions.

Let $P(t, x, u)$ be a probability density of joint distribution of random values $(X, U)$, subject to initial data

$$
P_{0}(x, u)=\delta\left(u-u_{0}(x)\right) \rho_{0}(x)=\prod_{k=1}^{n} \delta\left(u_{k}-\left(u_{0}(x)\right)_{k}\right) \rho_{0}(x),
$$

where $\rho_{0}(x)$ is a bounded nonnegative function from $L_{l o c}^{1}\left(\mathbb{R}^{n}\right)$.

Let us introduce functions

$$
\rho_{\sigma}(t, x)=\int_{\mathbb{R}^{n}} P(t, x, u) d u, \quad u_{\sigma}(t, x)=\frac{\int_{\mathbb{R}^{n}} u P(t, x, u) d u}{\int_{\mathbb{R}^{n}} P(t, x, u) d u} .
$$

Notice that $u_{\sigma}(0, x)=u_{0}(x)$. Certain properties of $u_{\sigma}(t, x)$ have been established in [2] and [3].

The density $P=P(t, x, u)$ obeys the Fokker-Planck equation

$$
\frac{\partial P}{\partial t}=\left[-\sum_{k=1}^{n} u_{k} \frac{\partial}{\partial x_{k}}+\sum_{k=1}^{n} \frac{1}{2} \sigma_{1}^{2} \frac{\partial^{2}}{\partial x_{k}^{2}}+\sum_{k=1}^{n} \frac{1}{2} \sigma_{2}^{2} \frac{\partial^{2}}{\partial u_{k}^{2}}\right] P,
$$

subject to initial data (1.3).

We apply the Fourier transform with respect to variables $x$ and $u$ in (1.5), (1.3) and obtain the following Cauchy problem for the Fourier transform $\tilde{P}=\tilde{P}(t, \lambda, \xi)$ of $P(t, x, u)$ :

$$
\begin{gathered}
\frac{\partial \tilde{P}}{\partial t}=-\frac{1}{2}\left(\sigma_{1}^{2}|\lambda|^{2}+\sigma_{2}^{2}|\xi|^{2}\right) \tilde{P}+\left(\lambda, \frac{\partial \tilde{P}}{\partial \xi}\right), \\
\tilde{P}(0, \lambda, \xi)=\int_{\mathbb{R}^{n}} e^{-i(\lambda, s)} e^{-i\left(\xi, u_{0}(s)\right)} \rho_{0}(s) d s, \quad \lambda, \xi \in \mathbb{R}^{n} .
\end{gathered}
$$

Equation (1.6) can easily be integrated and we obtain the solution:

$\tilde{P}(t, \lambda, \xi)=\tilde{P}(0, \lambda, \xi+\lambda t) \exp \left(-\frac{1}{2} \sigma_{1}^{2}|\lambda|^{2} t-\frac{\sigma_{2}^{2} t}{6}\left(|\lambda|^{2} t^{2}+3 t(\lambda, \xi)+3|\xi|^{2}\right)\right)$.

The inverse Fourier transform (in the distributional sense) allows to find the density $P(t, x, u)$ :

$$
P(t, x, u)=\frac{1}{(2 \pi)^{2 n}} \int_{\mathbb{R}^{n}} \int_{\mathbb{R}^{n}} e^{i(\lambda, x)} e^{i(\xi, u)} \tilde{P}(t, \lambda, \xi) d \lambda d \xi=
$$




$$
\begin{gathered}
=\frac{1}{(2 \pi)^{2 n}} \int_{\mathbb{R}^{n}} \rho_{0}(s) \int_{\mathbb{R}^{n}} \int_{\mathbb{R}^{n}} e^{-\frac{1}{2} \Lambda^{2}} e^{-\frac{1}{2} \Xi^{2}} e^{-\frac{\left|u_{0}(s)-u\right|^{2}}{2 t \sigma_{1}^{2}}-\frac{6\left|\left(u_{0}(s)+u\right) \frac{t}{2}+s-x\right|^{2}}{12 \sigma_{1}^{2} t+\sigma_{2}^{2} t^{3}}} d \lambda d \xi d s= \\
\quad=C \int_{\mathbb{R}^{n}} \rho_{0}(s) e^{-\frac{\left|u_{0}(s)-u\right|^{2}}{2 t \sigma_{1}^{2}}-\frac{6\left|\left(u_{0}(s)+u\right) \frac{t}{2}+s-x\right|^{2}}{12 \sigma_{1}^{2} t+\sigma_{2}^{2} t^{3}}} d s,
\end{gathered}
$$

where

$$
\begin{aligned}
& \Lambda=\Lambda(t, \lambda, s)=\frac{\sqrt{12 \sigma_{1}^{2} t+\sigma_{2}^{2} t^{3}}}{2 \sqrt{3}} \lambda+\frac{2 i \sqrt{3}}{\sqrt{12 \sigma_{1}^{2} t+\sigma_{2}^{2} t^{3}}}\left(\left(u_{0}(s)+u\right) \frac{t}{2}+s-x\right), \\
& \Xi=\Xi(t, \xi, s)=\sigma_{2} \sqrt{t} \xi+\frac{1}{\sigma_{2} \sqrt{t}}\left(\frac{\sigma_{2}^{2}}{2} \lambda t^{2}+i u_{0}(s)-u\right), \quad C=\left(\frac{\sqrt{3}}{\pi \sigma_{2} t \sqrt{12 \sigma_{1}^{2}+\sigma_{2}^{2} t^{2}}}\right)^{n} .
\end{aligned}
$$

From (1.9) we have

$$
\begin{aligned}
& \rho_{\sigma}(t, x)=\left(\frac{\sqrt{3}}{\left(\sqrt{2 \pi t\left(3 \sigma_{1}^{2}+\sigma_{2}^{2} t^{2}\right)}\right.}\right)^{n} \int_{\mathbb{R}^{n}} \rho_{0}(s) e^{-\frac{3\left|u_{0}(s) t+s-x\right|^{2}}{2 t\left(3 \sigma_{1}^{2}+\sigma_{2}^{2} t^{2}\right)}} d s, \\
& u_{\sigma}(t, x)=\frac{1}{2\left(3 \sigma_{1}^{2}+\sigma_{2}^{2} t^{2}\right)} \frac{\int_{\mathbb{R}^{n}} F(t, x, s) \rho_{0}(s) e^{-\frac{3\left|u_{0}(s) t+s-x\right|^{2}}{2 t\left(3 \sigma_{1}^{2}+\sigma_{2}^{2} t^{2}\right)}} d s}{\int_{\mathbb{R}^{n}} \rho_{0}(s) e^{-\frac{3\left|u_{0}(s) t+s-x\right|^{2}}{2 t\left(3 \sigma_{1}^{2}+\sigma_{2}^{2} t^{2}\right)}} d s},
\end{aligned}
$$

where $F(t, x, s)=6 \sigma_{1}^{2} u_{0}(s)-\sigma_{2}^{2} t\left(t u_{0}(s)+3(s-x)\right)$.

\section{Properties of the "Perturbed Velocity"}

Proposition 2.1. Let $u_{0}$ and $\rho_{0}>0$ be functions of class $C^{1}\left(\mathbb{R}^{n}\right) \cap$ $C_{b}\left(\mathbb{R}^{n}\right)$. If $t_{*}\left(u_{0}\right)>0$ is a moment of time such that the solution to the Cauchy problem (1.1) with the initial condition $u_{0}$ keeps this smoothness for $0<t<t_{*}\left(u_{0}\right) \leq+\infty$, then $\hat{u}_{\sigma}(t, x)$ tends to a solution of problem (1.1) as $|\sigma| \rightarrow 0$ for any fixed $(t, x) \in \mathbb{R}^{n+1}, 0<t<t_{*}\left(u_{0}\right)$.

Proof. Let us denote by $J\left(u_{0}(x)\right)$ the Jacobian matrix of the map $x \longmapsto$ $u_{0}(x)$. As it was shown in [4] (Theorem 1), if $J\left(u_{0}(x)\right)$ has at least one eigenvalue which is negative for a certain point $x \in \mathbb{R}^{n}$, then the classical solution to (1.1) fails to exist beyond a positive time $t_{*}\left(u_{0}\right)$. Otherwise, $t_{*}\left(u_{0}\right)=\infty$. The matrix $C(t, x)=\left(I+t J\left(u_{0}(x)\right)\right)$, where $I$ is the identity matrix, fails to be invertible for $t=t_{*}\left(u_{0}\right)$.

From (1.11) and (1.10) we have

$$
u_{\sigma}(t, x) \rho_{\sigma}(t, x)=\left(u_{\sigma}^{I}(t, x)-u_{\sigma}^{I I}(t, x)\right) \rho_{\sigma}(t, x),
$$


where

$$
\begin{gathered}
u_{\sigma}^{I}(t, x)=K \int_{\mathbb{R}^{n}} u_{0}(s) \rho_{0}(s) e^{-\frac{3\left|u_{0}(s) t+s-x\right|^{2}}{2 t\left(3 \sigma_{1}^{2}+\sigma_{2}^{2} t^{2}\right)}} d s, \\
u_{\sigma}^{I I}(t, x)=\frac{3 K \sigma_{2}^{2} t}{2\left(3 \sigma_{1}^{2}+\sigma_{2}^{2} t^{2}\right)} \int_{\mathbb{R}^{n}}\left(t u_{0}(s)+s-x\right) \rho_{0}(s) e^{-\frac{3\left|u_{0}(s) t+s-x\right|^{2}}{2 t\left(3 \sigma_{1}^{2}+\sigma_{2}^{2} t^{2}\right)}} d s, \\
K=\frac{\sqrt{3}}{\sqrt{2 \pi t\left(3 \sigma_{1}^{2}+\sigma_{2}^{2} t^{2}\right)}} \text {. Using the weak convergence of measures and }
\end{gathered}
$$
the fact that $\rho_{0}$ and $u_{0}$ are continuous and bounded, we get for $u_{\sigma}^{I}(t, x)$ :

$$
\begin{gathered}
\lim _{|\sigma| \rightarrow 0} u_{\sigma}^{I}(t, x)=\frac{\int_{\mathbb{R}^{n}} u_{0}(s) \rho_{0}(s) \lim _{\substack{\sigma_{1} \rightarrow 0 \\
\sigma_{2} \rightarrow 0}}\left(\frac{\sqrt{3}}{\sqrt{2 \pi t\left(3 \sigma_{1}^{2}+\sigma_{2}^{2} t^{2}\right)}}\right)^{n} e^{-\frac{3\left|u_{0}(s) t+s-x\right|^{2}}{2 t\left(3 \sigma_{1}^{2}+\sigma_{2}^{2} t^{2}\right)}} d s}{\int_{\mathbb{R}^{n}} \rho_{0}(s) \lim _{\substack{\sigma_{1} \rightarrow 0 \\
\sigma_{2} \rightarrow 0}}\left(\frac{\sqrt{3}}{\sqrt{2 \pi t\left(3 \sigma_{1}^{2}+\sigma_{2}^{2} t^{2}\right)}}\right)^{n} e^{-\frac{3\left|u_{0}(s) t+s-x\right|^{2}}{2 t\left(3 \sigma_{1}^{2}+\sigma_{2}^{2} t^{2}\right)}} d s} \\
=\frac{\int_{\mathbb{R}^{n}} u_{0}(s) \rho_{0}(s) \delta_{p(t, x, s)} d s}{\int_{\mathbb{R}^{n}} \rho_{0}(s) \delta_{p(t, x, s)} d s},
\end{gathered}
$$

with $p(t, x, s)=u_{0}(s) t+s-x$, where $\delta_{y}$ is the Dirac measure as $y \in \mathbb{R}^{n}$. We can then on the basis on the invertibility of $C(t, x)$ use locally the implicit function theorem and find $s=s_{t, x}(p)$.

Therefore,

$$
\begin{gathered}
\lim _{|\sigma| \rightarrow 0} u_{\sigma}^{I}(t, x)=\frac{\int_{\mathbb{R}^{n}} u_{0}\left(s_{t, x}(p)\right) \rho_{0}\left(s_{t, x}(p)\right) \operatorname{det}\left(C\left(t, s_{t, x}(p)\right)\right)^{-1} \delta_{p}\left(d s_{t, x}\right)}{\int_{\mathbb{R}^{n}} \rho_{0}\left(s_{t, x}(p)\right) \operatorname{det}\left(C\left(t, s_{t, x}(p)\right)\right)^{-1} \delta_{p}\left(d s_{t, x}\right)}= \\
=u_{0}\left(s_{t, x}(0)\right) .
\end{gathered}
$$

Now we prove that $\lim _{|\sigma| \rightarrow 0} u_{\sigma}^{I I}(t, x)=0$. We change variable $\tilde{s}=\frac{\sqrt{3}\left(u_{0}(s) t+s-x\right)}{\sqrt{t\left(3 \sigma_{1}^{2}+\sigma_{2}^{2} t^{2}\right)}}$ and obtain

$$
\lim _{|\sigma| \rightarrow 0} u_{\sigma}^{I I}(t, x)=\lim _{|\sigma| \rightarrow 0} \frac{\int_{\mathbb{R}^{n}} \sigma_{2}^{2} t \rho_{0}(\tilde{s}) \exp \left(-\tilde{s}^{2} / 2\right) d \tilde{s}}{\rho_{\sigma}(t, x)}=0 .
$$

Let us introduce the new notation $s_{0}(t, x) \equiv s_{t, x}(0)$. Then the following vectorial equation holds:

$$
u_{0}\left(s_{0}(t, x)\right) t+s_{0}(t, x)-x=0 .
$$

The function $u(t, x)=u_{0}\left(s_{0}(t, x)\right)$ satisfies the Burgers equation. To check this fact it is enough to differentiate (2.1) with respect to $t$ and 
$x_{j}$ and find $s_{0, t}$ and $s_{0, x}$. The more detailed proof you can find in [1] in Proposition 1.

It is important to note that $s_{0}(t, x)$ is unique for all $t$ for which the solution to the Burgers equation $u(t, x)$ is smooth.

Remark 2.2. Proposition 2.1 can naturally be extended to the class of functions $\rho_{0}$ such that there exists a sequence $\rho_{0}^{\varepsilon} \in C^{1}\left(\mathbb{R}^{n}\right) \cap C_{b}\left(\mathbb{R}^{n}\right)$ converging to $\rho_{0}$ as $\varepsilon \rightarrow 0$ almost everywhere. In this case $u_{\sigma}(t, x)$ tends to a solution of problem (1.1) as $\sigma \rightarrow 0$ almost everywhere on $(t, x) \in \mathbb{R}^{n+1}, 0<t<t_{*}\left(u_{0}\right)$.

Theorem 2.3. The scalar function $\rho_{\sigma}(t, x)$ and the vector-function $u_{\sigma}(t, x)$, defined in (1.4), solve the following system:

$$
\begin{gathered}
\frac{\partial \rho_{\sigma}}{\partial t}+\operatorname{div}_{x}\left(\rho_{\sigma} u_{\sigma}\right)=\frac{1}{2} \sigma_{1}^{2} \sum_{k=1}^{n} \frac{\partial^{2} \rho_{\sigma}}{\partial x_{k}^{2}} \\
\frac{\partial\left(\rho_{\sigma} u_{\sigma, i}\right)}{\partial t}+\operatorname{div}_{x}\left(\rho_{\sigma} u_{\sigma, i} u_{\sigma}\right)=\frac{1}{2} \sigma_{1}^{2} \sum_{k=1}^{n} \frac{\left.\partial^{2}\left(\rho_{\sigma} u_{\sigma, i}\right)\right)}{\partial x_{k}^{2}}-I_{\sigma}
\end{gathered}
$$

where $i=1, . ., n, t \geq 0$ and $I_{\sigma}=\int_{\mathbb{R}^{n}}\left(u_{i}-u_{\sigma, i}\right)\left(\left(u-u_{\sigma}\right), \nabla_{x} P(t, x, u)\right) d u$.

Proof. Integrating (1.5) with respect of $u$ we get:

$$
\frac{\partial \rho_{\sigma}}{\partial t}+\operatorname{div}_{x}\left(\rho_{\sigma} u_{\sigma}\right)=\frac{1}{2} \sigma_{1}^{2} \sum_{k=1}^{n} \frac{\partial^{2} \rho_{\sigma}}{\partial x_{k}^{2}}+\frac{1}{2} \sigma_{2}^{2} \sum_{k=1}^{n} \int_{\mathbb{R}^{n}} \frac{\partial^{2} P(t, x, u)}{\partial u_{k}^{2}} d u
$$

The last integral is equal to zero, because $P(t, x, u)$ and all its derivatives tend to zero as $|u| \rightarrow \infty$. Thus, we obtain (2.2).

To prove (2.3) we note that the definitions of $u_{\sigma}(t, x)$ and $\rho_{\sigma}(t, x)$ imply

$$
\begin{gathered}
\frac{\partial\left(\rho_{\sigma} u_{\sigma, i}\right)}{\partial t}=\frac{\partial}{\partial t} \int_{\mathbb{R}^{n}} u_{i} P d u=\int_{\mathbb{R}^{n}} u_{i} P_{t} d u=-\int_{\mathbb{R}^{n}} u_{i}\left(u, \nabla_{x} P\right) d u+ \\
\quad+\frac{1}{2} \sigma_{1}^{2} \sum_{k=1}^{n} \int_{\mathbb{R}^{n}} u_{i} \frac{\partial^{2} P}{\partial x_{k}^{2}} d u+\frac{1}{2} \sigma_{2}^{2} \sum_{k=1}^{n} \int_{\mathbb{R}^{n}} u_{i} \frac{\partial^{2} P}{\partial u_{k}^{2}} d u,
\end{gathered}
$$

where $P_{t} \equiv \frac{\partial}{\partial t} P$. Early we noted that the last integral is equal to zero.

Further, for $i=1, . ., n$ we have

$$
\operatorname{div}_{x}\left(\rho u_{\sigma, i} u_{\sigma}\right)=u_{\sigma, i} \operatorname{div}_{x}\left(\rho_{\sigma} u_{\sigma}\right)+u_{\sigma} \partial_{x}\left(\rho_{\sigma} u_{\sigma, i}\right)-u_{\sigma} u_{\sigma, i} \partial_{x} \rho_{\sigma}=
$$


$(2.5)=\int_{\mathbb{R}^{n}} u_{\sigma, i}\left(u, \nabla_{x} P\right) d u+\int_{\mathbb{R}^{n}} u_{i}\left(u_{\sigma}, \nabla_{x} P\right) d u-\int_{\mathbb{R}^{n}} u_{\sigma, i}\left(u_{\sigma}, \nabla_{x} P\right) d u$,

where $i, k=1, \ldots, n$. Equation (2.3) follows immediately from (2.4) and (2.5).

Let us set $\rho(t, x)=\lim _{|\sigma| \rightarrow 0} \rho_{\sigma}(t, x)$ and $\bar{u}(t, x)=\lim _{|\sigma| \rightarrow 0} u_{\sigma}(t, x)$.

Theorem 2.4. Assume that $(\rho(t, x), \bar{u}(t, x))$, the limits of $\left(\rho_{\sigma}, u_{\sigma}\right)$ as $|\sigma| \rightarrow 0$, are $C^{1}-$ smooth bounded functions for $(t, x) \in \Omega:=\left[0, t_{*}\left(u_{0}\right)\right) \times$ $\mathbb{R}^{n}, t_{*}\left(u_{0}\right) \leq \infty$. Then they solve in $\Omega$ the pressureless gas dynamics system

$$
\partial_{t} \rho+\operatorname{div}_{x}(\rho \bar{u})=0, \quad \partial_{t}(\rho \bar{u})+\nabla_{x}(\rho \bar{u} \otimes \bar{u})=0 .
$$

Proof. As follows from Proposition 2.1, the function $\bar{u}(t, x)$ is a $C^{1}$ solution of the non-viscous Burgers equation. Further, (2.2) is a linear parabolic equation with respect to $\rho_{\sigma}$, hence the limit as $|\sigma| \rightarrow 0$ reduces it to the first equation in (2.6) (continuity equation). The second equation in (2.6) is a corollary of the non-viscous Burgers equation and continuity equation for smooth solutions.

Remark 2.5. Proposition 2.4 implies that the integral term on the righthand side of (2.3) vanishes as $|\sigma| \rightarrow 0$ in the case of smooth limit functions $\rho$ and $\bar{u}$.

\section{A Regularization of THE PRESSURELESS GAS DYNAMiCS}

After the moment of shock formation in the solution to the Burgers equation the limit of (2.2)-(2.3) as $|\sigma| \rightarrow 0$ is not equivalent to the pressureless gas dynamics system (2.6), the integral term in (2.3) does not vanish and can be interpreted as a gradient of special pressure (spurious pressure). It is well known that for the pressureless gas dynamic system after the moment of singularity formation there arise a question how to define a generalized solution, and the answer depends on the prescribed type of interaction between particles. For the sticky particles model, where two particles move together after interaction, one need to introduce a strongly singular solution with a delta-singularity in the density component. If we assume that particles do not interact, we get the generalized solution in the sense of free particles, whose singularities are only shocks. In this sense we can call the limit of system (2.2)-(2.3) as $|\sigma| \rightarrow 0$ a regularization of the pressureless gas dynamics equations. There exist an algorithm how to pass from the model of free particles to the model of sticky particles (see [1] for details in the case $\left.\sigma_{2}=0\right)$. 
For discontinuous initial data analogously to [1] we introduce a generalized solution in the sense of free particles:

Definition 3.1. We call the couple of functions $\left(\rho_{F P}(t, x), u_{F P}(t, x)\right)$ the generalized solution to the Cauchy problem for (2.6) in the sense of free particles (FP-generalized solution) subject to initial data $\left(\rho_{0}(x), u_{0}(x)\right) \in \mathbb{L}_{l o c}^{2}\left(\mathbb{R}^{n}\right) \cap \mathbb{L}_{\infty}\left(\mathbb{R}^{n}\right)$, if for almost all $(t, x) \in \mathbb{R}_{+} \times \mathbb{R}^{n}$

$$
\rho_{F P}(t, x)=\lim _{\varepsilon \rightarrow 0}\left(\lim _{\sigma \rightarrow 0} \rho_{\sigma}^{\varepsilon}(t, x)\right), \quad u_{F P}(t, x)=\lim _{\varepsilon \rightarrow 0}\left(\lim _{\sigma \rightarrow 0} u_{\sigma}^{\varepsilon}(t, x)\right),
$$

where $\left(\rho_{\sigma}^{\varepsilon}(t, x), u_{\sigma}^{\varepsilon}(t, x)\right)$ correspond to initial data $\rho_{0}^{\varepsilon}=\eta_{\varepsilon} * \rho_{0}, \quad u_{0}^{\varepsilon}=$ $\eta_{\varepsilon} * u_{0}$, where $\eta_{\varepsilon}(x)$ is the standard averaging kernel.

In [1] for $\sigma_{2}=0$ we solve the Riemann problem in the sense of free particles, this can be done analogously in the general case $\sigma_{2}>0$.

It is very important that the double limit procedure used in the definition of the FP-solution plays role only for the case of a central rarefaction wave, where it helps to find a unique stable solution in the vacuum domain (we refer to [1] again). For the compression wave the FP-solution can be computed directly by formulae (1.10), (1.11) using only one limit pass $|\sigma| \rightarrow 0$. Thus, after the moment of the shock formation $t_{*}\left(u_{0}\right)$ in the Burgers equation the solution to the respective conservation law

$$
\partial_{t} \rho+\operatorname{div}_{x}(\rho \bar{u})=0, \quad \partial_{t}(\rho \bar{u})+\nabla_{x}(\rho \bar{u} \otimes \bar{u})+\nabla p=0, \quad \nabla p=\lim _{|\sigma| \rightarrow 0} I_{\sigma}
$$

can be found from (1.10), (1.11) as $|\sigma| \rightarrow 0$. By definition the solution to (3.1) is the FP-solution to (2.6), thus (3.1) can be considered as a regularization of (2.6).

\section{THE 1D MODEL FOR POLYTROPIC GAS}

Let us assume that the gas that obeys system (3.1) is polytropic, i.e. the state equation is $p=A \rho^{\gamma}, \gamma$ is the adiabatic exponent, $A$ is a positive constant. Therefore we have to supplement (3.1) with equation

$$
\partial_{t} p+u \partial_{x} p+\gamma p \partial_{x} u=0 .
$$

We consider the 1D Riemann problem in the compression case $u_{2}<0$ : $\rho_{0}(x)=\rho_{1}+\rho_{2} \theta(x), u_{0}(x)=u_{1}+u_{2} \theta(x)$, where $\rho_{i}, u_{i}, i=1,2$ are constants, In [1] the solution was obtained (for $\sigma_{2}=0$, the general case is analogous):

$$
\begin{gathered}
\rho(t, x)=\rho_{1}+\left(\rho_{1}+\rho_{2}\right) \theta\left(x-u_{1} t\right)-\rho_{1} \theta\left(x-\left(u_{1}+u_{2}\right) t\right), \\
u(t, x)=u_{1}+\frac{\rho_{1}+\rho_{2}}{2 \rho_{1}+\rho_{2}} u_{2} \theta\left(x-u_{1} t\right)-\frac{\rho_{1}}{2 \rho_{1}+\rho_{2}} u_{2} \theta\left(x-\left(u_{1}+u_{2}\right) t\right),
\end{gathered}
$$




$$
p(t, x)=\frac{\rho_{1}\left(\rho_{1}+\rho_{2}\right)}{2 \rho_{1}+\rho_{2}} u_{2}^{2}\left(\theta\left(x-\left(u_{1}+u_{2}\right) t\right)-\theta\left(x-u_{1} t\right)\right) .
$$

Proposition 4.1. In assumption that the state equation is polytropic, the adiabatic exponent $\gamma \rightarrow 3$ as the ratio $\frac{\rho_{1}}{\rho_{2}} \rightarrow 0$.

Proof. We have three Hugoniot conditions, that follow from the mass conservation, momentum conservation $((\underline{3.1}))$ and the conservation of total energy $E=\frac{1}{2} \rho u^{2}+\frac{p}{\gamma-1}$. We denote by $f_{-}$and $f_{+}$the values of $f(x)$ before and after shock, respectively. For the shock moving with the speed $D=u_{1}+u_{2}$ we have $\rho_{+}=2 \rho_{1}+\rho_{2}, \rho_{-}=\rho_{1}, u_{+}=u_{1}+$ $\frac{\rho_{1}+\rho_{2}}{2 \rho_{1}+\rho_{2}} u_{2}, u_{-}=u_{1}, p_{+}=\frac{\rho_{1}\left(\rho_{1}+\rho_{2}\right)}{2 \rho_{1}+\rho_{2}} u_{2}^{2}, p_{-}=0$. The third Hugoniot condition gives $D=\frac{[E u+p u]}{[E]}$ and $\gamma=\frac{3 \rho_{1}+\rho_{2}}{\rho_{1}+\rho_{2}}$. This implies the proposition. The consideration of the second shock gives the same result.

The adiabatic exponent $\gamma=1+\frac{2}{n}$, where $n$ is the space dimension, corresponds to the monoatomic gas.

\section{REFERENCES}

[1] S.Albeverio, A.Korshunova and O.Rozanova, Probabilistic model associated with the pressureless gas dynamics, submitted, arXiv:0908.2084.

[2] S.Albeverio and O.Rozanova, The non-viscous Burgers equation associated with random positions in coordinate space: a threshold for blow up behaviour, Math.Meth.Mod.Appl.Sci, 19 (2009), 1-19.

[3] S.Albeverio and O.Rozanova, Suppression of unbounded gradients in a SDE associated with the Burgers equation, Proc. Amer. Math. Soc. 138 (2010), 241-251.

[4] H.A.Levine and M.H.Protter, The breakdown of solutions of quasilinear first order systems of partial differential equations, Arch.Rat.Mech.Anal., 95 (1986), 253-267.

(1) Mathematics and Mechanics Faculty, Moscow State University, Moscow 119992, RussiA

E-mail address, ${ }^{1}$ : korshunova_aa@mail.ru

E-mail address, ${ }^{2}$ : rozanova@mech.math.msu.su 